\title{
Assessment of the responsiveness of four scales in geriatric patients with chronic neck pain
}

\author{
Mazlum Serdar Akaltun ${ }^{1}$ (1) $\cdot$ Burhan Fatih Kocyigit $^{2}$ (i)
}

Received: 23 June 2021 / Accepted: 19 July 2021 / Published online: 23 July 2021

(c) The Author(s), under exclusive licence to Springer-Verlag GmbH Germany, part of Springer Nature 2021

\begin{abstract}
The purpose of this study was to evaluate the responsiveness of Copenhagen Neck Functional Disability Scale (CNFDS), Neck Disability Index (NDI), Neck Bournemouth Questionnaire (NBQ), Neck Pain and Disability Scale (NPDS) in geriatric patients with non-specific chronic neck pain. A total of 52 geriatric patients with non-specific chronic neck pain were included in the present study. All participants were included in a physical therapy and rehabilitation program for five sessions per week that lasted 3 weeks. All participants were evaluated before and after the treatment. Pain and disability were assessed with the Visual Analog Scale-pain (VAS-pain), VAS-disability, muscle spasm, CNFDS, NDI, NBQ, NPDS scales. Additionally, patient satisfaction scores were questioned both before and after the treatment. Following the 3-week physical therapy and rehabilitation program, significant improvements were detected in the VAS-pain, VAS-disability, muscle spasm, CNFDS, NDI, NBQ, and NPDS scores $(p<0.05)$. Responsiveness values were found to be as follows: CNFDS [effect size $(\mathrm{ES})=0.78 ;$ standardized response mean $(\mathrm{SRM})=0.90]$, NDI $(\mathrm{ES}=0.66 ; \mathrm{SRM}=1.18), \mathrm{NBQ}(\mathrm{ES}=0.82 ; \mathrm{SRM}=0.97)$ and NPDS (ES $=0.87$; SRM =0.98). Our study demonstrated that CNFDS, NDI, NBQ, and NPDS are responsive scales in determining treatment-related changes in geriatric patients with non-specific neck pain. These results also suggest that all of these four scales can be used in the assessment of treatment induced changes in geriatric patients with chronic neck pain.
\end{abstract}

Keywords Responsiveness $\cdot$ Neck pain $\cdot$ Osteoarthritis $\cdot$ Rehabilitation $\cdot$ Geriatrics

\section{Introduction}

Neck pain is a common musculoskeletal system problem and is accepted as a substantial public health problem with its high socioeconomic effects $[1,2]$. It is estimated that the prevalence of neck pain is $40.5 \%$ in elderly women, and $36.1 \%$ in elderly men [3]. Osteoarthritis, which occurs as a result of degeneration in the cervical spine, is a considerable cause of chronic neck pain, and degeneration is observed particularly in the intervertebral disk and facet joints. It is clear that with increasing age, osteoarthritis-related changes

Burhan Fatih Kocyigit

bfk2701@hotmail.com

Mazlum Serdar Akaltun

mazlum_akaltun@hotmail.com

1 Faculty of Medicine, Department of Physical Medicine and Rehabilitation, Gaziantep University, Gaziantep, Turkey

2 Faculty of Medicine, Department of Physical Medicine and Rehabilitation, Kahramanmaraş Sütçü İmam University, Kahramanmaraş, Turkey in the cervical region become more pronounced and emerge as one of the main factors of chronic neck pain in the geriatric population [4]. Neck pain deteriorates physical and emotional conditions, loss of labor, and increased healthcare costs [5, 6]. Evaluation of pain and disability constitutes an important part of follow-up in patients with neck pain. There are many self-reporting scales assessing the pain and disability in this respect $[7,8]$. The assessment of the treatment outcomes is of great importance for clinicians and patients. In this context, self-reported scales are used widely to evaluate the treatment outcomes and follow-up patients. This kind of assessment also allows clinicians make evidence-based measurements and document the treatment process [9].

Self-reported outcome measures are an option that utilizes the patient view to evaluate the functional influence of a treatment protocol [10]. Responsiveness is defined as the ability of the measurement tool in detecting clinical changes [11]. The responsiveness value of a clinical measurement method is a key parameter for detecting changes resulting from a particular treatment. It is crucial for the preferred scales to have adequate responsiveness level to monitor the 
longitudinal change in a given treatment period. A responsive outcome measure should be capable of adequately reflecting clinically important change in patients' health conditions to guide treatment. Clinicians consider outcome measures when deciding whether to continue or change treatment protocols. Therefore, the responsiveness level of the scales becomes a cornerstone parameter in the followup studies and monitoring patients [12]. Although different methods have been suggested for responsiveness measurements, there is no gold standard method among them. Responsiveness is frequently assessed with the correlation analysis, effect sizes, standardized response means, sensitivity and specificity of change scores, relative efficiency, and receiver operating characteristics analysis [13].

It is considered that using criteria that are specific to neck pain instead of generic tools in evaluating the neck pain is more appropriate because of better responsiveness and better validity [14]. There are several studies in the literature investigating the responsiveness of different scales in patients with neck pain $[15,16]$. However, there are no studies conducted on geriatric patients with chronic neck pain. Given the particular differences of the geriatric population, we consider this to be an important deficiency. Responsiveness may vary according to the population, age, and etiology [17]. For this reason, the analyses must be specific to the population to predict responsiveness accurately [18]. Therefore, the aim of the present study was to evaluate the responsiveness of Copenhagen Neck Functional Disability Scale (CNFDS), Neck Disability Index (NDI), Neck Bournemouth Questionnaire (NBQ), and Neck Pain and Disability Scale (NPDS) in geriatric patients with chronic neck pain.

\section{Materials and methods}

\section{Study setting}

This is a descriptive pilot study. A total of 52 patients (36 females and 16 males) who had mechanical non-specific chronic neck pain admitting to the physical medicine and rehabilitation clinic between January 2020 and October 2020 were enrolled in the study. Informed consent was obtained from all patients. Approval for the study was granted by the Local Ethics Committee (approval date: 17.07.2019 and number: 08).

\section{Participants}

The inclusion criteria for the study were as follows: being between the ages of 65 and 80, complaining about neck pain for more than 3 months and consenting to participate in the study. Exclusion criteria were having a history of neck surgery, accompanying inflammatory rheumatic disorder, diseases causing neck pain, such as neoplasm, infection, abscess and neurological impairment, suffering prominent signs of radiculopathy and having a history of fractures or fixators in the cervical region. All patients were evaluated by the same physician with the anamnesis, physical examination. Past diseases of the participants were reviewed through hospital records. In addition, blood tests, magnetic resonance imaging, electromyography and X-rays of the patients were examined to confirm the exclusion criteria, if necessary.

\section{Demographic characteristics}

The sociodemographic and clinical characteristics, such as the age, sex, body mass index (BMI), educational status, working status, marital status, and disease duration of the patients, were questioned and recorded.

\section{Clinical evaluations}

In all patients, the pain was evaluated using the visual analog scale-pain (VAS-pain); and the patients were asked to give a score between $0-100 \mathrm{~mm}$ ( 0 point indicates no pain and 100 points indicate highest pain). Similarly, VAS was also used in evaluating the disability (VAS-disability), and the resulting scores were recorded.

The patients were asked to score muscle spasms between 0 (no spasms) and 6 (spasms at the highest level) to assess spasms in the muscles around the neck.

\section{Evaluation of patient satisfaction}

The patients were asked to score their treatment satisfaction on a five-point likert scale to assess patient satisfaction. According to this scale, one point indicates dissatisfaction, and five points indicate full satisfaction.

\section{Copenhagen Neck Functional Disability Scale}

CNFDS is a scale consisting of 15 items, and questions the impacts of neck pain. Each item is answered as "Yes" (0 points), "Sometimes" (1 point), and "No" ( 2 points). Questions 1 and 5 evaluate pain severity; items 2, 3, 4, 5, 7, 8, 9 and 10 question the difficulty in daily activities; and items 6 , $9,11,13$ and 14 question the social and recreational activities. Item 15 questions whether the neck pain will affect the future or not. The total score is between 0 and 30, and higher scores demonstrate greater disability [19]. The Turkish validity and reliability of the scale was conducted by Yapali et al. [20]. 


\section{Neck Disability Index}

NDI is a scale used frequently to measure neck pain-related disability. The scale was modified from Oswetry Disability Questionnaire. NDI consists of ten items, and each item is scored between 0 and 5 . Seven of these questions are about functional activities, 2 about symptoms, and 1 about concentration. The total score varies between 0 and 50. Higher scores indicate greater disability. Aslan et al. [21] performed the Turkish validity and reliability of the scale.

\section{Neck Pain and Disability Scale}

NPDS is a scale that was developed to evaluate neck pain in a multi-dimensional manner [22]. NPDS consists of 20 items, and assesses pain intensity, daily-life activities, and emotional-cognitive status. It is evaluated using evenlydivided VAS, and each item is scored between 0 and 5. The total score varies between 0 and 100 . The Turkish validity and reliability study of the scale was conducted by Bicer et al. [23].

\section{Neck Bournemouth Questionnaire}

NBQ consists of seven items, and questions pain severity, physical disability, social disability, anxiety, depression, employment, and pain control status [24]. Each item is scored between 0 and 10 . The Turkish validity and reliability of the scale was conducted by Yilmaz et al. [25].

\section{Physical therapy and rehabilitation protocol}

All the patients who were included in the study were taken to conventional physical therapy program for 3 weeks (5 days a week). Patients received 20 min hot-pack, 20 min transcutaneous electrical nerve stimulation (TENS) (TO ES-320, Chattanooga Intelect, USA), and 5 min continued ultrasound (BTL-4710 Sono Professional, BTL Medical Technologies Ltd, UK) (frequency, $1 \mathrm{MHz}$; intensity, $1 \mathrm{~W} / \mathrm{cm}^{2}$ ) in the conventional physical therapy program. In addition, in the content of the rehabilitation program, exercises to strengthen the deep neck muscles, isometric and isotonic neck exercises, stretching, relaxation and cervical stabilization exercises were performed by the same physiotherapist. It was started with the number of repetitions and difficulty level appropriate to the exercise background and physical condition of the patients. If the patient was appropriate, the exercise program was advanced. Rubber resistance bands were used during the isometric exercises. The resistance of the bands was gradually increased as the patient tolerated. Five low-resistance contractions were performed prior to the strengthening exercises followed by the predetermined number of repetitions at a difficulty of three-quarters of maximum capacity. This exercise program was planned and executed for 40 min daily [26].

\section{Comparisons}

All evaluations were performed by the same physician at the beginning and at the end of the physical therapy and rehabilitation program.

\section{Responsiveness measures}

Several methods have been used in responsiveness calculations; and there is no consensus on a gold standard method. The superiority of these methods over each other has not been demonstrated. The effect size (ES) and standardized response mean (SRM) values were calculated for the responsiveness evaluations of the four scales. The fact that it is widely used in the literature has been effective in our preference for this method. Thus, it was predicted that we would have the opportunity to compare the ES and SRM values in similar studies with our results. The ES value was obtained by dividing the mean of the change of baseline and follow-up scores by the standard deviation of the baseline score (Mean (Baseline - Follow-up scores) $\div$ SD - baseline score). The SRM value was calculated by dividing the mean of the change of baseline and follow-up scores by the standard deviation of the change score (Mean (Baseline - Follow-up scores) $\div$ SD - change score). Higher ES and SRM values indicate better responsiveness levels. Negativity shows that the baseline mean score was lower than the mean score in the follow-up, and this is related with the scale character. Therefore, negative values are not considered to be low responsiveness level. ES and SRM values are categorized as follows; values between 0.20 and 0.50 represent small responsiveness; values between 0.50 and 0.80 represent moderate responsiveness; and values equal to or higher than 0.80 represent large responsiveness [27-29].

\section{Statistical analyses}

Statistical analyses were performed using the Statistical Package for the Social Sciences version 20.0 package program (SPSS Inc., Chicago, IL, USA). The compliance of the data to normal distribution was checked using the Shapiro-Wilk test before the analyses. The expression of the quantitative data was carried out using the mean and standard deviation, and median (minimum-maximum) according to the distribution status. Number and percentage expressions were used in the categorical data. Paired $t$ test or Wilcoxon test was utilized to compare the scores measured before and after the physical therapy and rehabilitation program. The Spearman rho test was performed for correlation analyses. Significance value of the statistical analysis is 0.05 . 


\section{Results}

A total of 52 participants were included in the present study. Among these, $69.2 \%(n=36)$ were female, and $30.8 \%(n=16)$ were male; and the mean age was $70.19 \pm 3.69$ years. The socio-demographic and clinical characteristics of the participants are summarized in Table 1.

After the physical therapy and rehabilitation program, VAS-pain, VAS-disability, muscle spasm, CNFDS, NDI, NBQ and NPDS scores were significantly improved $(p<0.001)$ (Table 2).

After the physical therapy and rehabilitation program, significant correlations were detected between the patient satisfaction scores and CNFDS, NDI, NBQ and NPDS scores $(r=-0.677, p<0.001 ; r=-0.602, p<0.001$; $r=-0.533, p<0.001$ and $r=-0.634, p<0.001$, respectively) (Table 3 ).

Significant correlations were found between the changes in VAS-pain scores after the treatment and the changes in CNFDS, NDI, NBQ and NPDS scores $(r=0.434$, $p=0.001 ; r=0.574, p<0.001 ; r=0.587, p<0.001$ and $r=0.659, p<0.001)$. Additionally, significant correlations were determined between the changes in VAS-disability scores and the changes in CNFDS, NDI, NBQ and NPDS

Table 1 Baseline characteristics of the patients

\begin{tabular}{ll}
\hline $\begin{array}{l}\text { Age (years)* } \\
\text { Sex }[n(\%)]\end{array}$ & $70.19 \pm 3.69$ \\
Female & $36(69.2)$ \\
Male & $16(30.8)$ \\
BMI $^{+}$ & $27.34(21.56-40.44)(4.95)$ \\
Educational status $[n(\%)]$ & \\
$\quad$ Literate & $8(15.4)$ \\
Primary school & $24(46.2)$ \\
Middle School & $10(19.2)$ \\
High school & $8(15.4)$ \\
$\quad$ University or above & $2(3.8)$ \\
Working status $[n(\%)]$ & $4(7.7)$ \\
$\quad$ Working & $25(48.1)$ \\
Not-working & $23(44.2)$ \\
Retired & \\
Marital status $[n(\%)]$ & $29(55.8)$ \\
Married & $23(44.2)$ \\
Single or divorced & $48(3-120)(34.50)$ \\
Symptom duration (months) & +
\end{tabular}

$n$ Number, \% percentage, BMI body mass index

* Mean \pm standard deviation

${ }^{+}$Median (minimum-maximum) (interquartile range)

Table 2 Rehabilitation-induced changes in geriatric patients with neck pain

\begin{tabular}{llll}
\hline & Baseline & After rehabilitation & $p$ \\
\hline VAS-pain $^{+}$ & $80(50-100)$ & $60(50-90)$ & $<0.001$ \\
VAS-disability $^{+}$ & $80(30-100)$ & $60(50-90)$ & $<0.001$ \\
Muscle spasm $^{+}$ & $3(0-6)$ & $2(0-5)$ & $<0.001$ \\
CNFDS $^{*}$ & $21.25 \pm 4.66$ & $17.59 \pm 5.96$ & $<0.001$ \\
NDI $^{*}$ & $34 \pm 8.69$ & $28.19 \pm 10.70$ & $<0.001$ \\
NBQ $^{*}$ & $48.26 \pm 10.50$ & $39.55 \pm 14.26$ & $<0.001$ \\
NPDS $^{*}$ & $69.05 \pm 12.95$ & $57.73 \pm 19.58$ & $<0.001$ \\
\hline
\end{tabular}

VAS Visual Analog Scale, CNFDS Copenhagen Neck Functional Disability Scale, NDI Neck Disability Index, $N B Q$ Neck Bournemouth Questionnaire, NPDS Neck Pain and Disability Scale

*Mean \pm standard deviation

${ }^{+}$Median (minimum-maximum)

scores $(r=0.634, p<0.001 ; r=0.786, p<0.001 ; r=0.685$, $p<0.001$ and $r=0.759, p<0.001$, respectively).

ES and SRM values were calculated for the evaluation of responsiveness. The results demonstrated that the scales have moderate to large responsiveness levels in geriatric patients with chronic neck pain receiving physical therapy and rehabilitation program. Responsiveness values were found to be as follows: CNFDS ( $\mathrm{ES}=0.78$; $\mathrm{SRM}=0.90$ ), NDI $(\mathrm{ES}=0.66 ; \mathrm{SRM}=1.18)$, NBQ $(\mathrm{ES}=0.82 ; \mathrm{SRM}=0.97)$ and NPDS (ES $=0.87 ; \mathrm{SRM}=0.98)$ (Table 4).

\section{Discussion}

In the treatment of neck pain, the purpose is to reduce physical and emotional negative effects by ameliorating the pain and spasms, and also to improve the quality of life. Self-reported scales are substantial tools in determining the appropriate treatment strategy and monitoring treatment outcomes. In this regard, a scale to assess treatment response

Table 3 Correlations between scales after rehabilitation and Self-Assessment Patient Satisfaction Scores 
Table 4 Responsiveness values of the scales

\begin{tabular}{lll}
\hline Scales & ES & SRM $^{\mathrm{f}}$ \\
\hline CNFDS $^{\mathrm{a}}$ & 0.78 & 0.90 \\
$\mathrm{NDI}^{\mathrm{b}}$ & 0.66 & 1.18 \\
$\mathrm{NBQ}^{\mathrm{c}}$ & 0.82 & 0.97 \\
$\mathrm{NPDS}^{\mathrm{d}}$ & 0.87 & 0.98 \\
\hline
\end{tabular}

${ }^{\mathrm{a}}$ Copenhagen Neck Functional Disability Scale

${ }^{b}$ Neck Disability Index

${ }^{\mathrm{c}}$ Neck Bournemouth Questionnaire

${ }^{\mathrm{d}}$ Neck Pain and Disability Scale

${ }^{\mathrm{e}}$ Effect size

${ }^{\mathrm{f}}$ Standardized response mean

should be valid, reliable and responsive. This study demonstrated that each of the scales of CNFDS, NDI, NBQ, and NPDS have moderate to high responsiveness values in geriatric patients with chronic neck pain.

Responsiveness is almost as important as the validity and reliability for scales. Takeshita et al. [30] evaluated the validity, reliability, and responsiveness of NDI scale in patients with neck pain. They reported that the ES of the NDI and modified NDI scales to be 0.55 and 0.64 , respectively [30]. In previous studies, NDI had moderate-large responsiveness, which is similar to our study (0.81-1.17) [31]. Similarly, the SRM value of the NDI scale was found to be 0.85 in a study conducted with Turkish patients with non-geriatric and nonspecific neck pain [32]. The SRM value was found to be 1.18 in our study. The fact that this responsiveness values were obtained in the geriatric patient group suggests that NDI can be used in the assessment treatment response. NDI may be more useful in geriatric patients because it is relatively short and includes ten items. Items do not include VAS lines and offer five options. This is an important advantage taken into consideration the difficulty of geriatric population in completing long self-reported scales. Although not a long scale, NDI offers sufficient comprehensiveness in detecting changes in follow-up by questioning issues, such as personal care, headache, concentration, lifting, reading, working, driving, sleep, and entertainment, as well as pain intensity.

Stewart et al. [33] conducted a study on whiplash injury, and found that the ES value was 0.75 for the CNFDS scale, and the SRM was 0.90. Kose et al. [32] found SRM to be 0.89 in patients with chronic neck pain. In our study, ES was found to be 0.78, and SRM was 0.90, which suggest that CNFDS can also be used for follow-up of geriatric patients with neck pain. CNFDS does not have VAS lines, which some patients have difficulty in understanding. Although, its relatively long and includes 15 questions, each question has three options 'yes, occasionally or no'. This situation reduces the time required for the questions to be answered

and for the completion of the scale [19]. Having only three answer options avoids confusion in the geriatric population. Therefore, we consider that it can be used for the follow-up of geriatric patients with neck pain.

NPDS is another questionnaire used frequently for evaluating neck pain. NPDS questions pain intensity and daily-life activities along with emotional and cognitive status. Monticone et al. [34] found the SRM value as 1.26 in patients with chronic neck pain who admitted to the rehabilitation program. In a study conducted with Korean patients, the SRM value was found to be 1.34 for NPDS, and 1.17 for NDI [35]. In a study that was conducted in the Turkish population, the SRM value was found to be 0.92 [32]. The ES and SRM values were found to be 0.87 and 0.98 in geriatric patients with chronic neck pain in our study. Both values indicate that the scale is at the level of large responsiveness in this specific population. However, NPDS is the longest of these four scales and involves 20 items. All items are scored with the VAS line ranging from 0 to 5 points. This may cause confusion in the geriatric population and prevent the scale from being filled out accurately. Although the responsiveness values are large, we consider that NPDS should be clearly explained to the geriatric population before the utilization.

Gay et al. [24] found the SRM value of the NBQ questionnaire as 1.17, ES as 1.28; SRM value as 1.21, and ES as 1.12 for NDI in patients with chronic neck pain. They declared that both scales had better responsiveness compared to the changes in VAS. Stefanovitch-Lawbuary et al. [36] found that NBQ was more sensitive compared to the NDI, CNFDS, and Northwick Park Questionnaires in patients receiving physical therapy after whiplash injury. NBQ also showed large responsiveness in our study. NBQ also includes questions on pain and disability as well as psychosocial issues. The NBQ, the shortest of these four scales, consists of seven items. Considering that there may be a decrease in cognitive functions in the geriatric population, shortness becomes a very important advantage. However, scoring each item with the VAS line between 0 and 10 points can be a handicap. Taking into account the responsiveness values and scale features, NBQ can be considered as a preferable scale in the follow-up of geriatric patients with neck pain.

Changes in these four scales scores displayed significant correlations with changes in VAS-pain and VAS-disability scores. After the treatment, self-reported patient satisfaction scores significantly correlated with scores of these four scales. VAS-pain, VAS-disability and patient satisfaction scores are frequent parameters used by clinicians in clinical practice, and the correlations detected here support that these scales can be used both before and after the treatment.

Our study has several limitations. The relatively low sample size is one of them. The reasons for the low sample size were that we included only a subgroup of patients (geriatric patients) and strictly considered exclusion 
criteria. Also, we evaluated the patients only before and after the treatment. We did not assess the patients at longterm follow-up visits. Only geriatric patients with chronic neck pain were included in the study. Lack of evaluation of patients with acute and subacute neck pain may prevent generalization of the results. In addition, the results of our study are limited only with the Turkish population. Conducting similar studies in other countries is recommended. Besides all these limitations, substantial implications were obtained from the study. Our results have demonstrated that self-reported scales can be used in the follow-up of patients in geriatric chronic neck pain in which osteoarthritis is one of the main factors. In today's conditions, where we are actively struggling against COVID-19, online evaluations of patients have gained importance. Although we used the paper version of the scales in this study, the specified scales can be delivered to the patients online. As these scales are self-reported, patients can easily fill out and provide feedback on their condition without consulting the hospital. Considering that the geriatric population is in the risk group for COVID-19, it is a beneficial gain to evaluate and follow these patients with selfreported scales without applying to the hospital.

Rheumatic diseases can affect the musculoskeletal system and many other organs as a result of chronic inflammation. Considering that this group of diseases mostly sustains a chronic course and affects multiple organ systems, the importance of disability assessment becomes evident. It is a necessity that disability assessment is a part of follow-up processes not only in chronic pain syndromes but also in inflammatory rheumatic diseases. Disability can be evaluated with both anthropometric measurements and selfreported scales, and treatment protocols should be guided according to these results. Therefore, responsiveness studies of self-reported scales for disability should be conducted on rheumatic diseases and the most appropriate scales should be added to the follow-up processes.

As a conclusion, the results of the present study demonstrate that NDI, NPDS, CNFDS, and NBQ scales are responsive scales in geriatric patients with chronic neck pain. We consider that all four scales can be used in the follow-up of geriatric patients with neck pain. In addition to responsiveness values, clinicians should take into account the specific features of the scales and make their choices. Future studies should be planned with larger sample size and for longer follow-up periods.

Author contributions MSA and BFK designed the study. MSA and BFK collected the data. BFK analyzed the data. MSA and BFK interpreted the data analyses. MSA and BFK drafted the study and reviewed it critically for important intellectual content. BFK prepared tables. MSA and BFK approved the final version of the manuscript and agree to be accountable for all aspects of the work.
Funding The authors did not receive support from any organization for the submitted work.

\section{Declarations}

Conflict of interest There is no conflict interest among the authors.

Ethical approval Volunteerism was taken as the basis for participation and local ethics committee approved this study. This study conducted in compliance with Helsinki criteria.

Informed consent Informed consent was taken from the participants.

\section{References}

1. Kadhim-Saleh A, Maganti H, Ghert M, Singh S, Farrokhyar F (2013) Is low-level laser therapy in relieving neck pain effective? Systematic review and meta-analysis. Rheumatol Int 33:24932501. https://doi.org/10.1007/s00296-013-2742-z

2. Király M, Bender T, Hodosi K (2018) Comparative study of shockwave therapy and low-level laser therapy effects in patients with myofascial pain syndrome of the trapezius. Rheumatol Int 38:2045-2052. https://doi.org/10.1007/s00296-018-4134-x

3. March LM, Brnabic AJ, Skinner JC, Schwarz JM, Finnegan T, Druce J, Brooks PM (1998) Musculoskeletal disability among elderly people in the community. Med J Aust 168:439-442. https://doi.org/10.5694/j.1326-5377.1998.tb139023.x

4. Aslan Telci E, Karaduman A (2012) Effects of three different conservative treatments on pain, disability, quality of life, and mood in patients with cervical spondylosis. Rheumatol Int 32:10331040. https://doi.org/10.1007/s00296-010-1751-4

5. Koyuncu E, Ökmen BM, Özkuk K, Taşoğlu Ö, Özgirgin N (2016) The effectiveness of balneotherapy in chronic neck pain. Clin Rheumatol 35:2549-2555. https://doi.org/10.1007/ s10067-016-3199-8

6. Durmus D, Alayli G, Tufekci T, Kuru O (2014) A randomized placebo-controlled clinical trial of phonophoresis for the treatment of chronic neck pain. Rheumatol Int 34:605-611. https://doi.org/ 10.1007/s00296-013-2928-4

7. Saldaña MT, Navarro A, Pérez C, Masramón X, Rejas J (2010) Patient-reported-outcomes in subjects with painful lumbar or cervical radiculopathy treated with pregabalin: evidence from medical practice in primary care settings. Rheumatol Int 30:10051015. https://doi.org/10.1007/s00296-009-1086-1

8. Sutbeyaz ST, Sezer N, Koseoglu BF (2006) The effect of pulsed electromagnetic fields in the treatment of cervical osteoarthritis: a randomized, double-blind, sham-controlled trial. Rheumatol Int 26:320-324. https://doi.org/10.1007/s00296-005-0600-3

9. Renskers L, van Uden RJJC, Huis AMP, Rongen SAA, Teerenstra S, van Riel PLCM (2018) Comparison of the construct validity and reproducibility of four different types of patient-reported outcome measures (PROMs) in patients with rheumatoid arthritis. Clin Rheumatol 37:3191-3199. https://doi.org/10.1007/ s10067-018-4285-x

10. Çelik D, Malkoç M, Martin R (2016) Evidence for reliability, validity and responsiveness of Turkish Foot and Ankle Ability Measure (FAAM). Rheumatol Int 36:1469-1476. https://doi.org/ 10.1007/s00296-016-3485-4

11. Moon KW, Lee SS, Kim JH, Song R, Lee EY, Song YW, Bellamy N, Lee EB (2012) Cross-cultural adaptation, validation, and responsiveness of the Korean version of the AUSCAN 
Osteoarthritis Index. Rheumatol Int 32:3551-3557. https://doi. org/10.1007/s00296-011-2227-x

12. Salaffi F, Stancati A, Carotti M (2002) Responsiveness of health status measures and utility-based methods in patients with rheumatoid arthritis. Clin Rheumatol 21:478-487. https://doi.org/10. 1007/s100670200119

13. Sezer N, Yavuzer G, Sivrioglu K, Basaran P, Koseoglu BF (2007) Clinimetric properties of the Duruoz hand index in patients with stroke. Arch Phys Med Rehabil 88:309-314. https://doi.org/10. 1016/j.apmr.2006.12.019

14. Riddle DL, Stratford PW (1998) Use of generic versus regionspecific functional status measures on patients with cervical spine disorders. Phys Ther 78:951-963. https://doi.org/10.1093/ptj/78.9. 951

15. Jorritsma W, Dijkstra PU, de Vries GE, Geertzen JH, Reneman MF (2012) Detecting relevant changes and responsiveness of Neck Pain and Disability Scale and Neck Disability Index. Eur Spine J 21:2550-2557. https://doi.org/10.1007/s00586-012-2407-8

16. Young BA, Walker MJ, Strunce JB, Boyles RE, Whitman JM, Childs JD (2009) Responsiveness of the Neck Disability Index in patients with mechanical neck disorders. Spine J 9:802-808. https://doi.org/10.1016/j.spinee.2009.06.002

17. DeVellis RF (1996) A consumer's guide to finding, evaluating, and reporting on measurement instruments. Arthritis Care Res 9:239-245. https://doi.org/10.1002/1529-0131(199606)9:3\% 3c239::aid-anr1790090313\%3e3.0.co;2-o

18. Young IA, Dunning J, Butts R, Mourad F, Cleland JA (2019) Reliability, construct validity, and responsiveness of the neck disability index and numeric pain rating scale in patients with mechanical neck pain without upper extremity symptoms. Physiother Theory Pract 35:1328-1335. https://doi.org/10.1080/09593 985.2018.1471763

19. Angilecchia D, Mezzetti M, Chiarotto A, Daugenti A, Giovannico G, Bonetti F (2018) Development, validity and reliability of the Italian version of the Copenhagen neck functional disability scale. BMC Musculoskelet Disord 19:409. https://doi.org/10. 1186/s12891-018-2332-z

20. Yapali G, Günel MK, Karahan S (2012) The cross-cultural adaptation, reliability, and validity of the Copenhagen Neck Functional Disability Scale in patients with chronic neck pain: Turkish version study. Spine (Phila Pa 1976) 37:E678-E682. https://doi.org/ 10.1097/BRS.0b013e31824b549c

21. Aslan E, Karaduman A, Yakut Y, Aras B, Simsek IE, Yaglý N (2008) The cultural adaptation, reliability and validity of neck disability index in patients with neck pain: a Turkish version study. Spine (Phila Pa 1976) 33:E362-365. https://doi.org/10.1097/BRS. 0b013e31817144e1

22. Wheeler AH, Goolkasian P, Baird AC, Darden BV (1999) Development of the Neck Pain and Disability Scale. Item analysis, face, and criterion-related validity. Spine (Phila Pa 1976) 24:12901294. https://doi.org/10.1097/00007632-199907010-00004

23. Bicer A, Yazici A, Camdeviren H, Erdogan C (2004) Assessment of pain and disability in patients with chronic neck pain: reliability and construct validity of the Turkish version of the neck pain and disability scale. Disabil Rehabil 26:959-962. https://doi.org/10. 1080/09638280410001696755

24. Gay RE, Madson TJ, Cieslak KR (2007) Comparison of the Neck Disability Index and the Neck Bournemouth Questionnaire in a sample of patients with chronic uncomplicated neck pain. J Manip Physiol Ther 30:259-262. https://doi.org/10.1016/j.jmpt.2007.03. 009
25. Y1lmaz O, Gafuroğlu Ü, Yüksel S (2018) Translation, reliability, and validity of the Turkish version of the Neck Bournemouth Questionnaire. Turk J Phys Med Rehabil 65:59-66. https://doi. org/10.5606/tftrd.2019.2693

26. Thompson DP, Oldham JA, Woby SR (2016) Does adding cognitive-behavioural physiotherapy to exercise improve outcome in patients with chronic neck pain? A randomised controlled trial. Physiotherapy 102:170-177. https://doi.org/10.1016/j.physio. 2015.04.008

27. De Smet L (2004) Responsiveness of the DASH score in surgically treated basal joint arthritis of the thumb: preliminary results. Clin Rheumatol 23:223-224. https://doi.org/10.1007/ s10067-004-0866-y

28. Traki L, Rostom S, Tahiri L, Bahiri R, Harzy T, Abouqal R, Hajjaj-Hassouni N (2014) Responsiveness of the EuroQol EQ-5D and Hospital Anxiety and Depression Scale (HADS) in rheumatoid arthritis patients receiving tocilizumab. Clin Rheumatol 33:10551060. https://doi.org/10.1007/s10067-014-2609-z

29. Kocyigit BF, Akaltun MS (2020) Assessment of responsiveness of four hand-related scales in stroke patients. Acta Neurol Belg. https://doi.org/10.1007/s13760-020-01443-x

30. Takeshita K, Hosono N, Kawaguchi Y, Hasegawa K, Isomura T, Oshima Y, Ono T, Oshina M, Oda T, Kato S, Yonenobu K (2013) Validity, reliability and responsiveness of the Japanese version of the Neck Disability Index. J Orthop Sci 18:14-21. https://doi.org/ 10.1007/s00776-012-0304-y

31. Vernon H (2008) The Neck Disability Index: state-of-the-art, 1991-2008. J Manip Physiol Ther 31:491-502. https://doi.org/ 10.1016/j.jmpt.2008.08.006

32. Kose G, Hepguler S, Atamaz F, Oder G (2007) A comparison of four disability scales for Turkish patients with neck pain. J Rehabil Med 39:358-362. https://doi.org/10.2340/16501977-0060

33. Stewart M, Maher CG, Refshauge KM, Bogduk N, Nicholas M (2007) Responsiveness of pain and disability measures for chronic whiplash. Spine (Phila Pa 1976) 32:580-585. https://doi.org/10. 1097/01.brs.0000256380.71056.6d

34. Monticone M, Ambrosini E, Vernon H, Brunati R, Rocca B, Foti C, Ferrante S (2015) Responsiveness and minimal important changes for the Neck Disability Index and the Neck Pain Disability Scale in Italian subjects with chronic neck pain. Eur Spine J 24:2821-2827. https://doi.org/10.1007/s00586-015-3785-5

35. Lee H, Nicholson LL, Adams RD, Maher CG, Halaki M, Bae SS (2006) Development and psychometric testing of Korean language versions of 4 neck pain and disability questionnaires. Spine (Phila Pa 1976) 31:1841-1845. https://doi.org/10.1097/01.brs.00002 27268.35035.a5

36. Stefanovitch-Lawbuary N, Amirfeyz R, Lovell R, Bannister G (2019) Reliability and responsiveness of patient-reported outcome measures of neck disability to physical therapy: comparison of the Copenhagen, Northwick Park, and Neck Bournemouth Questionnaires and the Neck Disability Index. J Manip Physiol Ther 42:104-107. https://doi.org/10.1016/j.jmpt.2019.03.007

Publisher's Note Springer Nature remains neutral with regard to jurisdictional claims in published maps and institutional affiliations. 